\title{
TRATAMENTO DA DESNUTRIÇÃO EM CRIANÇAS HOSPITALIZADAS EM SÃO PAULO
}

\author{
Rosel Oselka Saccardo Sarni*, Fabíola Isabel Suano De Souza, Priscila Catherino, Cristiane Kochi, \\ Fernanda luisa Ceragioli Oliveira, Fernando José De Nóbrega \\ Trabalho realizado no Núcleo de Nutrição, Alimentação e Desenvolvimento Infantil do Centro de \\ Referência de Saúde da Mulher, Nutrição, Alimentação e Desenvolvimento Infantil da Secretaria de \\ Estado da Saúde de São Paulo, São Paulo, SP.
}

RESUMO - OвjEtivo. Avaliar a evolução antropométrica, terapia nutricional e mortalidade de crianças desnutridas hospitalizadas em centro de referência.

Métodos. Em estudo retrospectivo, avaliou-se 98 prontuários de crianças desnutridas $(\mathrm{ZPI}<-2)$, sem doença crônica associada. Dados coletados: diagnóstico à internação (DI), tipo, via e tolerância da dieta, tempo de internação (TINT). Peso e estatura na internação e na alta. Para classificação antropométrica e evolução nutricional utilizou-se: índices peso/estatura (ZPE), estatura/idade (ZE) e peso/idade (ZP) expressos na forma de escore Z. A terapia utilizada baseou-se nas normas da Organização Mundial da Saúde (OMS) com adaptações em relação à dieta. Empregou-se sempre fórmulas industrializadas: infantil polimérica isenta de lactose (FI) para crianças com diarréia, com menor conteúdo de lactose (FB) para crianças sem diarréia e infantil semi-elementar contendo proteína do leite de vaca hidrolisada até peptídios $(\mathrm{H})$ para crianças em sepse ou diarréia crônica (fase de estabilização). Para todas as crianças na fase de recuperação nutricional utilizou-se fórmula com menor conteúdo de lactose (FB). Estudo estatístico: teste t-pareado e Qui-quadrado.

Resultados. Encontrou-se mediana de idade de 9,8 meses, TINT 17 dias e taxa de letalidade de $2 \%$. Pneumonia e doença diarréica corresponderam a $81,6 \%$ dos DI. Observou-se melhora do ZE, ZP e ZPE em $17,3 \%$, 82,7\% e $92,2 \%$ das crianças, respectivamente. Dieta mais utilizada na admissão foi a $\mathrm{Fl}(47,4 \%)$ sendo a fórmula $\mathrm{H}$ utilizada em $7,4 \%$ das crianças. $\mathrm{Na}$ evolução da terapia nutricional, constatou-se uso de sonda em $20,4 \%$ e nutrição parenteral em 5, $1 \%$ das crianças. Em relação à tolerância à dieta, observou-se que $87,8 \%$ apresentaram boa evolução com a dieta inicialmente instituída.

Conclusöes. 0 protocolo modificado da OMS foi efetivo no tratamento de crianças gravemente desnutridas, propiciando recuperação nutricional satisfatória com baixo índice de letalidade.

Unitermos: Desnutrição. Internamento. Terapia nutricional. Diretrizes OMS. Crianças.

\section{INTRODUÇÃO}

Tem sido observada na literatura uma significativa redução na prevalência da desnutrição energético-protéica (DEP) em diversas partes do mundo, incluindo o Brasil 1,2. Apesar disso, tal doença ainda se configura como importante problema saúde pública, especialmente em crianças menores de cinco anos.

Ressaltam-se, entretanto, os elevados índices de letalidade, inalterados nas últimas décadas, ocorrendo especialmente nas formas graves de desnutrição (mediana de $20 \%$ a $30 \%$ entre as décadas de 50 a 90) $)^{3}$. Uma das causas prováveis para esse fato reside no desconhecimento dos profissionais de saúde com relação à fisiopatologia da DEP grave e, conse-

*Correspondência:

Rua René Zamlutti, 94 apto. 52

CEP: $04116-260$ - São Paulo/SP

E-mail: rssarni@uol.com.br

Fone/fax: (11) 5571-9589 qüentemente, da instituição de uma terapia inadequada que resulta em sérias complicações logo nos primeiros dias da internação hospitalar, culminando com a morte.

Tendo em vista a necessidade de atualização e adequada capacitação dos profissionais de saúde envolvidos na assistência a crianças gravemente desnutridas, a Organização Mundial de Saúde (OMS) publicou e divulgou, em 1999, um manual com essa finalidade ${ }^{4}$. Essa publicação divide em três etapas o tratamento do desnutrido (estabilização, reabilitação e seguimento), que devem transcorrer por aproximadamente 26 semanas. Em todas as fases, leva-se em conta as características fisiopatológicas peculiares da DEP grave e a importância da abordagem multidisciplinar, visando dessa forma tratar a criança de forma global, com a participação da família, evitando recorrências e reduzindo a mortalidade 5 .

Ainda há poucos estudos na literatura avaliando as repercussões da utilização do protocolo da $\mathrm{OMS}^{6}$, mas os resultados iniciais são muito favoráveis, inclusive em relação à mortalidade ${ }^{7,8}$

Tendo em vista a complexidade do atendimento ao desnutrido grave, especialmente, na fase inicial (intra-hospitalar) e a escassez de estudos publicados na literatura abordando tal tema, foi realizado esse trabalho, que teve como objetivo avaliar a evolução antropométrica, terapia nutricional e letalidade de crianças desnutridas hospitalizadas, sem doença crônica associada, em centro de referência (Núcleo de Nutrição, Alimentação e Desenvolvimento Infantil do Centro de Referência de Saúde da Mulher, Nutrição, Alimentação e Desenvolvimento Infantil da Secretaria de Estado da Saúde de São Paulo - NUNADI/ CRSMNADI/SES), utilizando-se as práticas propostas no manual da OMS.

\section{MÉTodos}

Em estudo retrospectivo foram avaliadas, entre janeiro/99 e outubro/00, a totalidade das internações que ocorreram no NUNADI/ 
CRSMNADI. A Unidade de Internação do NUNADI fornecia atendimento multiprofissional a crianças de 0 a 5 anos, portadoras de desnutrição com descompensação infecciosa e/ou ocasionada por distúrbios hidroeletrolíticos e ácido-básicos. As crianças desnutridas eram sempre referenciadas de hospitais da rede pública de saúde e, após a alta hospitalar, permaneciam sob assistência da equipe em regime ambulatorial ou de hospital-dia.

Foram levantados 305 prontuários, dos quais 98 foram selecionados. Os critérios utilizados para seleção dos pacientes foram z escore de peso para idade ZPS -2 à admissão hospitalar e ausência de doença crônica associada (cardiopatia, neuropatia, hepatopatia, Aids, imunodeficiências congênitas, síndromes genéticas, endocrinopatia e nefropatia).

Por meio de questionário padronizado, foram levantados os seguintes dados dos prontuários selecionados: peso ao nascer (PN), idade gestacional $(I G)$, aleitamento materno exclusivo ou predominante (AME/P), diagnóstico à internação (DI), tipo de dieta e via de administração, tolerância à dieta, uso de nutrição parenteral, tempo de internação (TINT) e evolução do estado nutricional (melhorado, piorado ou inalterado).

O peso era obtido por intermédio de balança tipo plataforma para crianças com $>15 \mathrm{~kg}$ (precisão de $100 \mathrm{~g}$ ) e em balança tipo pesa-bebê para crianças com $<15 \mathrm{~kg}$ (precisão de $5 g$ ). Para aferição da estatura utilizava-se estadiômetro vertical ou horizontal de madeira, graduado em milímetros, segundo preconização de Jellife?.

A partir dos dados de peso e estatura calculou-se os índices antropométricos, expressos na forma de z escore: índices peso para idade (ZP), estatura para idade (ZE) e peso para estatura (ZPE) ${ }^{10}$. A evolução do estado nutricional foi obtida subtraindo-se o z escore (ZP, ZE, ZPE) da saída e o da entrada. A partir dessa diferença considerou-se a evolução do estado nutricional como: melhorado $(+)$, piorado (-) ou inalterado (0).

A terapia nutricional utilizada baseou-se nas normas propostas no protocolo da OMS para 0 tratamento de crianças desnutridas ${ }^{4}$, com algumas adaptações em relação ao tipo da dieta utilizada, porém sempre com o emprego de fórmulas industrializadas: fórmula infantil polimérica isenta de lactose (FI) para crianças com diarréia, com menor conteúdo de lactose (FB - 35\% do total de carboidratos) para crianças sem diarréia, e fórmula infantil semi-elementar contendo proteína do leite de vaca hidrolisada até peptídios $(H)$ para crianças com sepse ou diarréia crônica como dieta inicial (fase de estabilização) e FB para todas as crianças na fase de recuperação nutricional. As condutas propostas pela OMS foram referendadas em publicações recentes, em nosso meio, realizadas pelo Ministério da Saúde, Organização Pan-americana da Saúde, Sociedade Brasileira de Pediatria e Universidades $11,12,13$.

A oferta energética utilizada foi de 80 a $100 \mathrm{kcal} / \mathrm{kg} / \mathrm{dia}$ na fase de estabilização e 150 a $200 \mathrm{kcal} / \mathrm{kg} / \mathrm{dia}$ na fase de recuperação nutricional ${ }^{4}$. Quando necessário, para respeitar a oferta hídrica máxima de 130 $\mathrm{ml} / \mathrm{kg} / \mathrm{dia}$, na fase inicial, utilizou-se módulos de triglicérides de cadeia média (TCM) em crianças com doença diarréica, ou de cadeia longa ( $T C L)$ em crianças sem doença diarréica, acrescidos à dieta na proporção máxima de 3\%; e/ou módulos de polímeros de glicose (máximo 3\%). $\mathrm{Na}$ fase de recuperação nutricional utilizouse 0 acréscimo de $3 \%$ de TCL (óleo de soja) à dieta visando adequação da densidade energética $(0,93 \mathrm{kcal} / \mathrm{ml})$.

A troca da dieta, $\mathrm{FB} \rightarrow \mathrm{Fl}$ ou Fl $\rightarrow \mathrm{H}$, foi considerada quando ocorriam manifestações de intolerância (diarréia, vômitos, distensão abdominal, dermatite perineal) e/ ou evolução ponderal desfavorável. A administração da dieta por sondas ocorreu quando a ingestão era inferior ao gasto energético basal acrescido a fator de estresse de $20 \%$ a $30 \%$ (<80 kcal $/ \mathrm{kg} / \mathrm{dia}$ ), quando havia risco de aspiração pulmonar ou em casos em intolerância alimentar. A dieta por sondas era iniciada após estabilização hidroeletrolítica visando reduzir complicações como a síndrome de realimentação ${ }^{12}$.

Todas as crianças receberam suplementação de vitaminas na forma medicamentosa incluindo: ácido fólico (I mg/dia), ferro (3 mg/kg/dia -após estabilização) na forma de sulfato ferroso, zinco (2 mg/kg/dia) e cobre $(0,2 \mathrm{mg} / \mathrm{kg} / \mathrm{dia})$ na forma de módulo acrescido à dieta. As quantidades oferecidas de vitaminas e oligoelementos seguiram as preconizadas no manual da OMS4.
Para análise estatística utilizou-se os testes do Qui-quadrado para comparação entre as variáveis qualitativas, $t$ de Student pareado para comparação entre as médias do z escore (entrada e saída). Adotou-se como 0,05 ou 5\% o nível de rejeição para a hipótese de nulidade assinalando-se com asteriscos os valores significantes.

\section{Resultados}

A caracterização da população estudada está disposta na Tabela I. Predominou na amostra o sexo masculino (58,2\%), detectou-se uma grande proporção de alterações no período neonatal: baixo peso ao nascer em $49,3 \%$, comprimento ao nascer inferior a $47 \mathrm{~cm}$ em $77,8 \%$, prematuridade em 33,8\% e intercorrências pós-parto imediatas em $25,7 \%$ da população (Tabela I). Nota-se também que 0 tempo de aleitamento materno predominante foi bem inferior ao recomendado, alcançando a mediana de I,I 5 meses.

Apenas dois pacientes (2\%) evoluíram para óbito, ambos ocasionados por sepse. Os diagnósticos mais freqüentes à admissão foram a pneumonia e a doença diarréica, englobando cerca de $81,6 \%$ das causas de internação (Tabela 2).

A mediana de idade à internação foi de 9,8 meses e a do tempo de internação 17 dias. Houve um evidente comprometimento dos índices antropométricos à admissão: mediana do ZPE, ZP e ZE; -2,I (-3,8; I,0); $3,22(-6,8 ;-2,1)$; e $-2,93(-8,9 ;-0,5)$, respectivamente. Nenhuma criança apresentou edema nutricional.

Quando se estudou a evolução dos índices antropométricos considerando a diferença entre alta e admissão, constatou-se uma melhora mais evidente do ZPE (92,2\%), seguido pelo ZP (82,7\%) e, finalmente, pelo ZE (I7,3\%) (Gráfico I). A mediana do ganho de peso foi de 6 gramas $/ \mathrm{kg} / \mathrm{dia}$. Houve melhora estatisticamente significante dos índices ZPE e ZP, e piora do ZE (Tabela 3).

A dieta mais utilizada à admissão foi a FI (47,4\%), sendo a dieta $\mathrm{H}$ utilizada apenas em 7,4\% dos pacientes. A distribuição da dieta utilizada na população avaliada, relacionando a evolução antropométrica está disposta na Tabela 4. 


\begin{tabular}{|c|c|c|}
\hline \multicolumn{3}{|c|}{ Tabela I - Características da população estudada } \\
\hline VARIÁVEL & $\mathbf{N}$ & $\%$ \\
\hline \multicolumn{3}{|l|}{ Sexo } \\
\hline Feminino & 41 & $41,8 \%$ \\
\hline Masculino & 57 & $58,2 \%$ \\
\hline \multicolumn{3}{|l|}{ Peso nascimento } \\
\hline$\leq 2500 \mathrm{~g}$ & 37 & $49,3 \%$ \\
\hline$>2500 \mathrm{~g}$ & 38 & $50,7 \%$ \\
\hline \multicolumn{3}{|l|}{ Comprimento nascimento } \\
\hline$\leq 47 \mathrm{~cm}$ & 35 & $77,8 \%$ \\
\hline$>47 \mathrm{~cm}$ & 10 & $22,2 \%$ \\
\hline \multicolumn{3}{|l|}{ Idade gestacional } \\
\hline Pré-termo & 29 & $33,3 \%$ \\
\hline Termo & 58 & $66,7 \%$ \\
\hline \multicolumn{3}{|l|}{ Intercorrências neonatais } \\
\hline Hipóxia & II & $15,7 \%$ \\
\hline Infecção & 4 & $5,7 \%$ \\
\hline Tocotrauma & 3 & $4,3 \%$ \\
\hline \multicolumn{3}{|c|}{ Tempo de aleitamento materno (exclusivo/predominante) } \\
\hline$\leq 1$ mês & 26 & $34,8 \%$ \\
\hline $\mid \neg 3$ meses & 17 & $22,7 \%$ \\
\hline $3 \neg 6$ meses & 8 & $10,7 \%$ \\
\hline Nuncamamou & 23 & $30,8 \%$ \\
\hline \multicolumn{3}{|l|}{ Evolução } \\
\hline Alta & 96 & $98 \%$ \\
\hline Óbito & 2 & $2 \%$ \\
\hline
\end{tabular}

\begin{tabular}{lcc}
\hline \multicolumn{3}{c}{ Tabela 2 - Diagnósticos mais freqüentes à internação } \\
\hline VIA & $\mathbf{N}$ & $\%$ \\
Pneumonia & 31 & $31,6 \%$ \\
Doença diarréica ${ }^{*}$ & 49 & $50 \%$ \\
Baixo ganho ponderal & 8 & $8,2 \%$ \\
Anemia & 4 & $4,1 \%$ \\
Outros & 6 & $6,1 \%$ \\
Total & 98 & $100 \%$ \\
\hline
\end{tabular}

\# Inclui doença diarréica aguda e persistente

Gráfico I - Percentual de melhora e piora das crianças segundo o delta (saída - entrada) do ZPE $(\mathrm{N}=64), Z P(\mathrm{~N}=97)$ e ZE $(\mathrm{N}=75)$

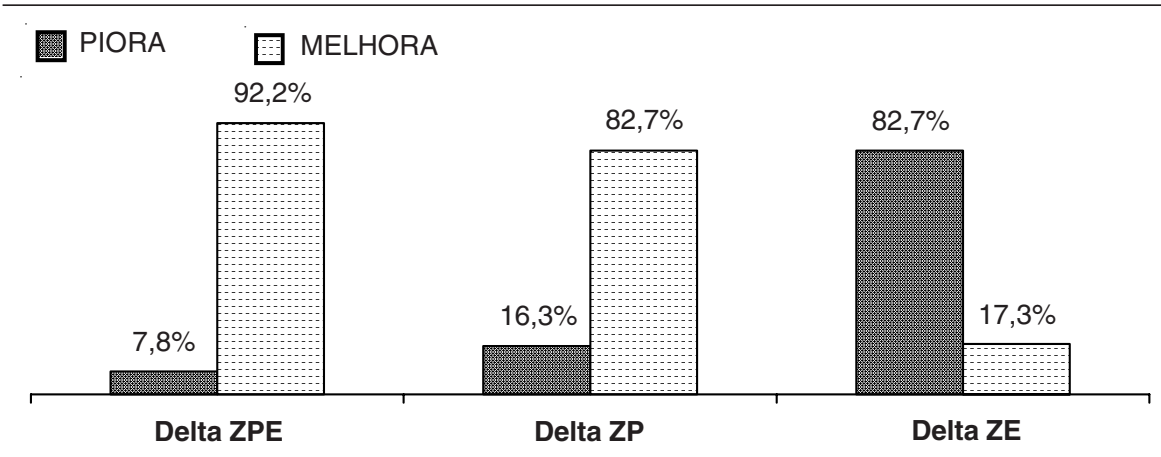

Delta ZPE: diferença entre o z escore peso/estatura na alta e na admissão; Delta ZE: diferença entre o z escore estatura/idade na alta e na admissão; Delta ZP: diferença entre o z escore peso/idade na alta e na admissão
A via inicial mais freqüente de administração da dieta foi a oral (88,8\%), seguida pelo uso de sonda nasogástrica ou póspilórica (I0,2\%). A nutrição parenteral foi utilizada inicialmente em apenas $1 \%$ das crianças avaliadas (Tabela 5).

Apesar de somente 10\% das crianças utilizarem a sonda como via inicial para administração da dieta, no decorrer da internação esse número praticamente dobrou 20/98 $(20,4 \%)$, sendo a causa mais freqüente da modificação da via de administração a ingestão calórica insuficiente e a intolerância à dieta por via oral I6/20 (80\%). Já em relação à nutrição parenteral, apenas 5/98 (5,1\%) das crianças evoluíram para essa forma de terapia nutricional.

Em relação à tolerância à dieta, observase que a maioria dos pacientes evoluiu satisfatoria-mente com a dieta inicialmente instituída 60/90 (66,7\%). Considerou-se evolução desfavorável da dieta inicialmente prescrita quando houve progressão para fórmulas mais complexas $(\mathrm{Fl} \rightarrow \mathrm{H}$ ou FB $\rightarrow \mathrm{FI}$ ) e evolução favorável quando a evolução ocorreu de forma inversa $(\mathrm{H} \rightarrow \mathrm{FB}$ ou $\mathrm{Fl} \mathrm{e} \mathrm{Fl} \rightarrow$ $F B)$. Levando-se em conta esses critérios, 30/ 90 (33,3\%) dos pacientes foram submetidos à troca de fórmula, sendo que 19/30 (63,3\%) por evolução favorável e II/30 (36,7\%) por evolução desfavorável (Tabela 6).

\section{Discussão}

A desnutrição não é resultado da carência exclusiva de alimentos, é na realidade multifatorial envolvendo aspectos de natureza médica e social que têm as suas raízes na pobreza. Tal modelo foi descrito por Nóbrega \& Campos em 1996, que destacaram entre os diversos fatores envolvidos na gênese da DEP o fraco vínculo mãe-filho ${ }^{15}$.

Frente a esse modelo multicausal da DEP fica claro que o seu combate ultrapassa os limites da abordagem médica, seja em nível primário ou terciário de atenção à saúde e requer, para gerar resultados positivos, o envolvimento de equipe multiprofissional preparada para lidar com as particularidades do atendimento ao desnutrido e sua família. Com essa finalidade foi criado em |991, pela Secretaria do Estado da Saúde de São Paulo, o NUNADI, que apresentava como objetivos básicos o atendimento 
Tabela 3 - Evolução antropométrica dos pacientes durante a internação, relacionada aos índices antropométricos na admissão e na alta

\begin{tabular}{|c|c|c|c|c|c|}
\hline \multirow{2}{*}{$\begin{array}{l}\text { Variável } \\
\text { ZPEadmissão } \\
\text { ZPE alta }\end{array}$} & & \multirow{2}{*}{$\begin{array}{l}\frac{N}{68} \\
66\end{array}$} & \multicolumn{2}{|c|}{ MÉDIA DP } & \multirow{2}{*}{$\frac{\text { Valor-p }}{<0,000 \mid *}$} \\
\hline & & & $\begin{array}{r}-1,90 \\
-0,94\end{array}$ & $\begin{array}{l}( \pm 1,06) \\
( \pm 1,35)\end{array}$ & \\
\hline $\begin{array}{l}\text { ZEadmissão } \\
\text { ZE alta }\end{array}$ & Delta ZPE & $\begin{array}{l}64 \\
78 \\
74\end{array}$ & $\begin{array}{l}0,93 \\
-3,25 \\
-3,46\end{array}$ & $\begin{array}{l}( \pm 0,96) \\
( \pm 1,87) \\
( \pm 1,76)\end{array}$ & $0,001 *$ \\
\hline $\begin{array}{l}\text { ZP admissão } \\
\text { ZP alta }\end{array}$ & Delta ZEI & $\begin{array}{l}75 \\
98 \\
98\end{array}$ & $\begin{array}{l}-0,16 \\
-3,47 \\
-3,04\end{array}$ & $\begin{array}{l}( \pm 0,59) \\
( \pm 1,01) \\
( \pm 1,00)\end{array}$ & $<0,\left.000\right|^{\prime}$ \\
\hline & Delta ZPI & 98 & 0,43 & $( \pm 0,57)$ & \\
\hline
\end{tabular}

Teste t-pareado (Alta - Admissão)

ZPE Z escore peso/estatura

ZE $\quad$ z escore estatura/idade

ZP $\quad$ z escore peso/idade

Tabela 4 - Distribuição do tipo da dieta utilizada à admissão, relacionada com a evolução da condição nutricional (ZPE, ZE e ZP na Alta - Admissão)

\begin{tabular}{|c|c|c|c|c|c|c|c|c|}
\hline \multirow[t]{2}{*}{ Dieta } & & & \multicolumn{2}{|c|}{ Delta ZPE } & \multicolumn{2}{|c|}{ Delta ZE } & \multicolumn{2}{|c|}{ Delta ZP } \\
\hline & & & $(+)$ & $(-)$ & $\overline{(+)}$ & $(-)$ & $\overline{(+)}$ & $(-)$ \\
\hline \multirow[t]{2}{*}{$H$} & $\mathrm{~N}$ & 7 & 3 & - & I & 4 & 5 & 2 \\
\hline & $\%$ & $7,4 \%$ & $100 \%$ & - & $20 \%$ & $80 \%$ & $71,4 \%$ & $28,6 \%$ \\
\hline \multirow[t]{2}{*}{$\mathrm{Fl}$} & $\mathrm{N}$ & 45 & 25 & | & 9 & 23 & 38 & 7 \\
\hline & $\%$ & $47,4 \%$ & $96 \%$ & $4 \%$ & $28,1 \%$ & $71,9 \%$ & $84,5 \%$ & $15,5 \%$ \\
\hline \multirow[t]{2}{*}{$F B$} & $N$ & 43 & 30 & 3 & 2 & 33 & 36 & 6 \\
\hline & $\%$ & $45,3 \%$ & $91 \%$ & $9 \%$ & $5,7 \%$ & $94,3 \%$ & $85,7 \%$ & $14,3 \%$ \\
\hline Total & $\mathrm{N}$ & 95 & 58 & 4 & 12 & 60 & 79 & 15 \\
\hline
\end{tabular}

Evolução antropométrica:

Delta ZPE, ZE e ZP ( $(+)$ Melhorado

Delta ZPE, ZE e ZP (-) Piorado

Delta ZPE diferença entre o $z$ escore peso/estatura na alta e na admissão

Delta ZE diferença entre $\mathrm{z}$ z escore estatura/idade na alta e na admissão

Delta ZP diferença entre $0 \mathrm{z}$ escore peso/idade na alta e na admissão

- Dieta:

H fórmula semi-elementar contendo proteína do leite de vaca hidrolisada até peptídeos

$\mathrm{Fl} \quad$ fórmula infantil polimérica isenta de lactose

FB fórmula infantil polimérica com menor conteúdo de lactose

\begin{tabular}{|c|c|c|c|c|c|c|c|c|}
\hline \multirow[t]{2}{*}{ Via } & & & & & & & & ZP \\
\hline & & & $\overline{(t)}$ & $(-)$ & $\overline{(+)}$ & $(-)$ & $(+)$ & $(-)$ \\
\hline Oral & $\begin{array}{l}N \\
\%\end{array}$ & $\begin{array}{l}87 \\
88,8 \%\end{array}$ & $\begin{array}{l}54 \\
91,5 \%\end{array}$ & $\begin{array}{l}5 \\
8,5 \%\end{array}$ & $13,5 \%$ & $\begin{array}{l}58 \\
86,5 \%\end{array}$ & $\begin{array}{l}73 \\
85 \%\end{array}$ & 13 \\
\hline Sonda & $\begin{array}{l}N \\
\%\end{array}$ & $\begin{array}{l}10 \\
10,2 \%\end{array}$ & $\begin{array}{l}4 \\
100 \%\end{array}$ & - & $\begin{array}{l}3 \\
42,8 \%\end{array}$ & $\begin{array}{l}4 \\
57,2 \%\end{array}$ & $\begin{array}{l}7 \\
70 \%\end{array}$ & $\begin{array}{l}3 \\
30 \%\end{array}$ \\
\hline $\begin{array}{l}\text { Parenteral } \\
\text { TOTAL }\end{array}$ & $\begin{array}{l}N \\
\% \\
N\end{array}$ & $1,0 \%$ & $\begin{array}{l}1 \\
100 \% \\
59\end{array}$ & $\frac{-}{5}$ & $\begin{array}{l}1 \\
100 \% \\
13\end{array}$ & $\frac{-}{62}$ & $\begin{array}{l}1 \\
100 \% \\
81\end{array}$ & $\frac{-}{16}$ \\
\hline
\end{tabular}

Evolução antropométrica:

Delta ZPE, ZE e ZP (+) Melhorado

Delta ZPE, ZE e ZP (-) Piorado

Delta ZPE diferença entre $0 \mathrm{z}$ escore peso/estatura na alta e na admissão

Delta ZE diferença entre $0 \mathrm{z}$ escore estatura/idade na alta e na admissão

Delta ZP diferença entre o $\mathrm{z}$ escore peso/idade na alta e na admissão aos distúrbios da nutrição em regime ambulatorial, hospital-dia e internação.

Visando conhecer melhor a população atendida neste Núcleo, do ponto de vista nutricional, realizou-se esse levantamento de todas as internações realizadas nos anos de 1999 e 2000 , após a implantação do protocolo proposto pela OMS para tratamento da DEP grave ${ }^{4}$.

A taxa de letalidade encontrada nesse estudo foi de $2 \%$, evidenciando a importância do atendimento da criança gravemente desnutrida em centro de referência especializado. A OMS considera o índice de letalidade como um dos indicadores que refletem o sucesso ou a falência do atendimento ao desnutrido em unidades hospitalares. Valores superiores a $20 \%$ são considerados inaceitáveis, I1\%-20\% letalidade moderada, 5\%-10\% letalidade baixa, 1\%$4 \%$ bom índice de letalidade e $<1 \%$ excelente índice de letalidade ${ }^{16}$.

As variáveis mais fortemente relacionadas à mortalidade na admissão de uma criança com DEP grave são: a gravidade da condição nutricional, presença de edema nutricional, deficiência de micronutrientes, desbalanço hidroeletrolítico, disfunção hepática e infecções associadas, além da não utilização de um protocolo de tratamento ${ }^{17}$. Em estudo longitudinal na comunidade de Guiné-Bissau, Perra \& Costello encontraram uma redução de $25 \%$ na mortalidade e melhora da evolução nutricional de crianças gravemente desnutridas com a utilização de um protocolo de atendimento simples, respaldado na fisiopatologia da DEP, a um custo aceitável ${ }^{18}$.

Em estudo conduzido em Bangladesh com 437 crianças gravemente desnutridas, de 12 a 60 meses, verificou-se recuperação nutricional ( $P / E>80 \%)$ em regime de internação, hospital-dia e domiciliar. Os autores não encontraram diferença na mortalidade (inferior a 5\% nos três grupos), o custo/dia do paciente internado foi superior aos outros dois grupos, contudo o tempo para atingir recuperação nutricional foi significativamente menor no grupo de pacientes internados ${ }^{19}$.

O tempo de recuperação é uma variável importante no tratamento; a OMS preconiza que se atinja a relação peso/estatura (PE) superior a $90 \%$ no período de 6 semanas, pois 


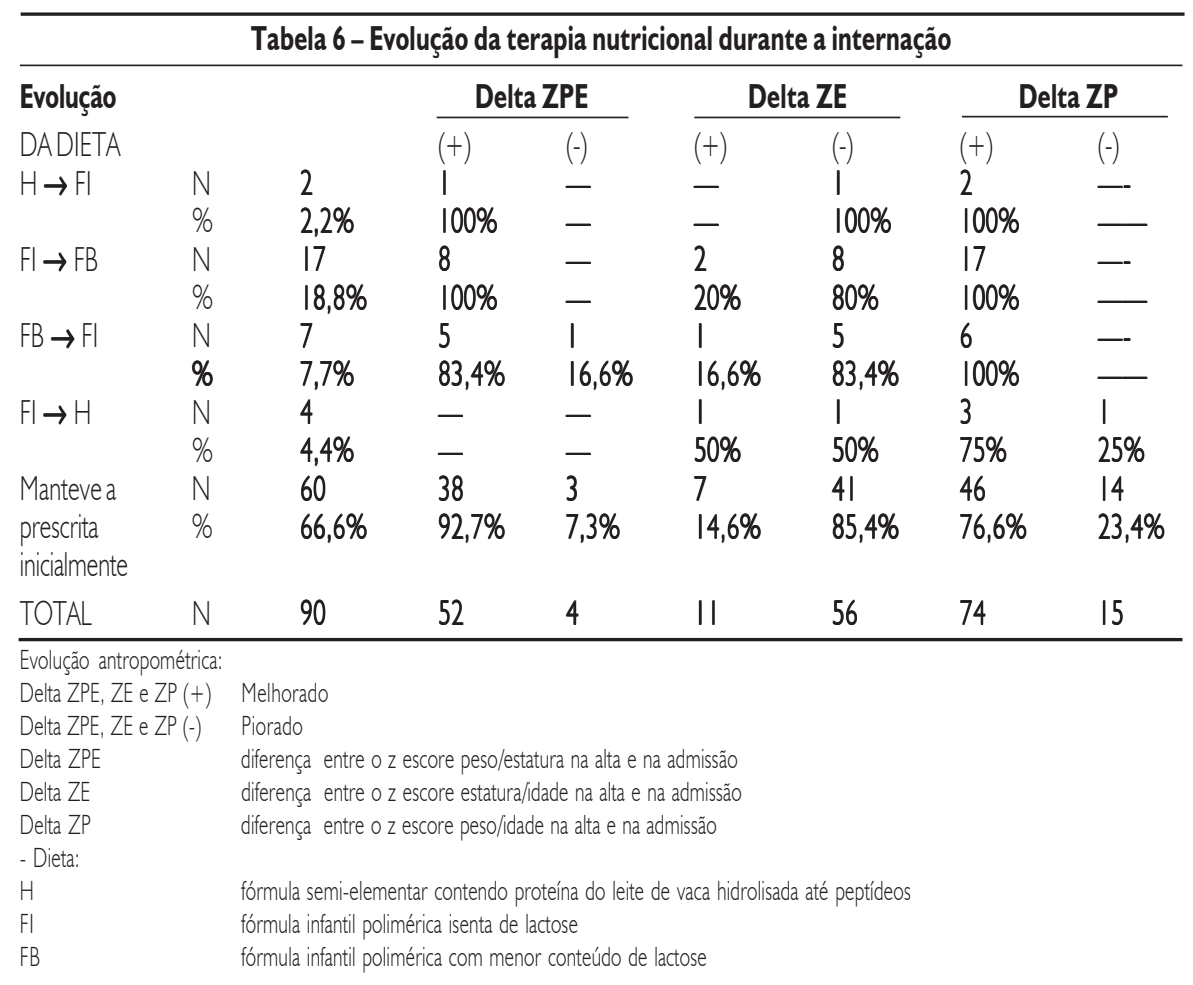

sabe-se que a demora em atingir essa meta incorre em aumento da mortalidade e eleva o número de seqüelas.

No presente estudo, verificou-se melhora da condição nutricional considerando os índices ZPE e ZP na população avaliada, com uma mediana de 17 dias de internação. A mediana de ganho ponderal (6 g/kg/dia) foi compatível com a considerada adequada pela OMS que preconiza um ganho mínimo de $5 \mathrm{~g} / \mathrm{kg} / \mathrm{dia}^{4}$.

$\mathrm{O}$ índice estatura para idade (ZE) revelou uma recuperação menos evidente; somente 17,5\% das crianças melhoraram o ZE. Sabe-se que a recuperação estatural é mais lenta comparada à de peso, por esse motivo, a OMS considera apenas a recuperação PE como critério de alta hospitalar. Embora mais lenta, sua monitorização é fundamental no seguimento ambulatorial posterior à alta hospitalar, uma vez que o déficit estatural conseqüente à DEP relaciona-se com várias seqüelas na vida adulta, incluindo maior incidência de doenças crônicas não transmissíveis e pior desempenho cognitivo futuro ${ }^{20,21}$.

Vale a pena ressaltar ainda, que encontrou-se na população avaliada elevado percentual de baixo peso ao nascer $(49,3 \%)$ que constitui importante fator de risco para o crescimento estatural deficiente, principalmente nos dois primeiros anos de vida ${ }^{22}$, podendo se perpetuar para a adolescência e idade adulta ${ }^{23,24}$.

Em relação ao diagnóstico à admissão, a doença diarréica foi a principal causa de internação seguida pela infecção pulmonar aguda. Estudo de Rice et al., encontrou uma forte e consistente relação entre desnutrição e risco de morte por diarréia e infecção respiratória aguda ${ }^{25}$; além disso, quanto maior o grau de desnutrição, maior foi o risco de morte, o que torna importante o diagnóstico e tratamento precoces das intercorrências infecciosas, uma vez que o desnutrido é imunocomprometido ${ }^{26}$.

A abordagem aos distúrbios hidroeletrolíticos em crianças gravemente desnutridas também foi revista pela OMS, que recomenda o uso de solução de reidratação adaptada (maior conteúdo de potássio e menor de sódio, acrescida de micronutrientes), menores volumes para hidratação intravenosa, uma vez que o reconhecimento de sinais de desidratação é difícil nessas crianças. Ressalta-se também os cuidados com a oferta excessiva de sódio e vigilância estreita do potássio e magnésio séricos ${ }^{8}$. Essas práticas foram adotadas em todas as crianças atendidas no NUNADI que apresentavam distúrbios hidroeletrolíticos.

Nenhuma criança apresentou forma edematosa de DEP grave, durante o período de estudo. A forma marasmo de DEP grave predomina nas regiões Sul e Sudeste do Brasil, enquanto que as regiões Norte e Nordeste ainda apresentam elevada freqüência de formas kwashiorkor ${ }^{27}$.

Um dos grandes avanços no conhecimento da fisiopatologia e tratamento $\mathrm{da}$ DEP nas últimas décadas foi a respeito da gênese do edema, antes atribuída exclusivamente à hipoalbuminemia. Nesse sentido, Golden propôs a participação do estresse oxidativo como um dos mecanismos responsáveis pela alteração na permeabilidade capilar e conseqüente edema na DEP gra$v^{28-31}$. Essa abordagem trouxe novas perspectivas para o seu tratamento, especialmente em relação à importância da suplementação de micronutrientes ${ }^{32}$.

A dieta mais utilizada à admissão foi a fórmula industrializada polimérica isenta de lactose (FI). Estudos mostram que a utilização de dietas com baixo teor de lactose são benéficas na recuperação nutricional de crianças desnutridas. Na DEP há uma redução da atividade das enzimas utilizadas na digestão dos açúcares, especialmente a lactase ${ }^{33,34}$.

No Manual da OMS, tanto a dieta para a fase de estabilização (F-75) quanto para a fase de reabilitação possuem baixo conteúdo de lactose, I,3 e 4,2 g/l00mL. No NUNADI, além da fórmula isenta de lactose, utilizou-se também dieta polimérica com baixo teor de lactose (FB) nas crianças sem doença diarréica e na fase de recuperação nutricional (2,6 g/ $100 \mathrm{~mL}$ ) para reduzir o risco de intolerância.

Utilizou-se dieta semi-elementar $(\mathrm{H})$ apenas em 7,4\% das crianças. Não há evidências na literatura de benefícios na utilização de hidrolisado em crianças gravemente desnutridas. Em estudo recente, os autores compararam o uso de duas fórmulas isentas de lactose, com osmolalidades diferentes ( 65 vs 200 mOsm/kg água) e hidrolisado protéico no tratamento da diarréia em crianças desnutridas. Encontrou-se um maior tempo de duração dos episódios diarréicos com o uso do hidrolisado em relação às fórmulas poliméricas. $\bigcirc$ ganho ponderal foi 
superior nas crianças que utilizaram a fórmula polimérica isenta de lactose com menor osmolalidade ${ }^{35}$.

Em relação à tolerância à dieta, observou-se que 79/90 (87,8\%) das crianças apresentaram boa evolução com a dieta inicialmente prescrita. Em II/90 (I2,2\%), optou-se pela troca de dieta tendo em vista algumas intercorrências como: ganho de peso inadequado ou manifestações de intolerância. A evolução favorável com a terapia nutricional proposta reflete a importância do planejamento da conduta terapêutica. Com respeito ao fornecimento energético, salienta-se que a oferta excessiva de nutrientes (hiperalimentação), especialmente em crianças desnutridas em fase aguda de processo infeccioso, pode levar à hiperglicemia, hiperosmolalidade, diurese osmótica, desidratação, esteatose hepática e hipertrigliceridemia ${ }^{36,37}$.

Não foi possível, neste estudo, utilizar a dieta preconizada pela OMS devido à dificuldade na produção do mix de minerais e vitaminas e, por esse motivo, utilizaram fórmulas industrializadas. Estudos recentes mostram que a utilização das dietas propostas pela OMS resultam em expressiva redução nos custos do tratamento de crianças gravemente desnutridas ${ }^{7}$.

$A$ via enteral foi significativamente mais utilizada do que a parenteral durante toda a internação. Indicou-se o uso de sondas, inicialmente, em 10\% das crianças, após estabilização hidroeletrolítica, contudo, durante a evolução, esse percentual praticamente dobrou para $20,4 \%$.

A causa mais freqüente para indicação da sonda foi a oferta energética insuficiente na fase inicial (inferior a $80 \mathrm{kcal} / \mathrm{kg} / \mathrm{dia}$ ), uma vez que a anorexia é bastante comum, especialmente nas fases iniciais do tratamento das crianças gravemente desnutridas. $\mathrm{O}$ uso da sonda nesta fase pode garantir a manutenção da condição nutricional. Entretanto, é fundamental que previamente à infusão da dieta por sonda os eletrólitos séricos estejam normalizados. Tal procedimento evita a síndrome de realimentação, fenômeno presumivelmente causado pelo deslocamento de eletrólitos intracelulares ocasionado pela utilização de glicose e produção de adenosina trifosfato ${ }^{37}$.

\section{Conclusão}

Publicações recentes com a aplicação do protocolo proposto pela OMS demonstram resultados satisfatórios, assim como os encontrados nesse estudo, incluindo a redução substancial nos índices de letalidade por desnutrição grave em crianças. Experiências positivas nessa redução já foram observadas também em Bangladesh, África do Sul e no Brasil (Ceará). A facilidade de implantação do referido protocolo, que pode ser efetuada em cerca de uma semana - desde a avaliação dos problemas existentes na Unidade Hospitalar, treinamento da equipe de profissionais e implementação da unidade - justificam sua utilização em larga escala em nosso meio ${ }^{7}$.

$\bigcirc$ atendimento à criança desnutrida por equipe multiprofissional em centros especializados, com padronização de condutas adaptadas à fisiopatologia destes pacientes, como a proposta pela OMS, promove a recuperação nutricional, com tempo de internação satisfatório e baixo índice de letalidade. Conclui-se, ainda, que é de fundamental importância o seguimento após a alta hospitalar, visto que a recuperação estatural é mais tardia.

\section{Conflito de interesse: não há.}

\section{SUMMARY}

\section{NUtRITIONAL SUPPORT FOR MALNOU- RISHED HOSPITALIZED CHILDREN: EXPE- rience of a referral center, São Paulo, BRAZIL}

Alm. Tostudy anthropometric development, nutritional support and mortality rate of malnourished children hospitalized in a referral center

METHODS. In a retrospective study we surveyed 98 hospitalized malnourished children $(Z W<-2)$ with no chronic disease. Data collected was: birth weight, gestational age, length of exclusive breast feeding, diagnosis at admission, formula used (type, delivery route and feeding tolerance) and length of stay. Weight and height were controlled at admission and discharge. To classify and evaluate nutritional rehabilitation we used the Z-score: weight-for-age $(Z W)$, height-for-age $(Z H)$, weight-for-height $(Z \mathrm{~W} / \mathrm{H})$. The nutritional therapy used was based on the World Health Organization (WHO) guidelines, with minor modifications. All chosen formulas were industrialized: lactose-free polymeric formula (PLF) for children with diarrhea, low lactose polymeric formula (PLL) for children without diarrhea and cow's milk hydrolysate (CMH) for sepsis or chronic diarrhea. At the rehabilitation phase, all children were given the PL L formula.

Statistical analysis. Student's $t$ and chisquare tests.

REsULTS. The median of age and length of stay were 9.8 months and 17 days, respectively and the mortality rate was of $2 \%$. Diarrhea and/ or pneumonia were diagnosed at admission in $81.6 \%$ of the children. An improvement of 17.3 $\% \mathrm{ZW}, 82.7 \% \mathrm{ZH}$ and $92.2 \% \mathrm{ZW} / \mathrm{H}$ was observed. PLF was more frequently given at admission (47.4\%) while CMH was given to only 7.4\% of the children. Twenty-fourpercent of the children were tube fed and $5.1 \%$ received parenteral nutrition. Tolerance of the initial formula was considered good in $66.7 \%$ of cases.

Conclusions. The WHO guidelines were effective in the nutritional therapy of severely malnourished hospitalized children, resulting in good nutritional rehabilitation with low mortality rates. [Rev Assoc Med Bras 2005; 5 I (2): I06- I 2]

KEY WORDS: Malnutrition. Hospitalization. Nutritional support. WHO guidelines. Children.

\section{RefERÊNCIAS}

I. United Nations Children's Foundation. The state of the world's children 2002. New York: Unicef; 2002.

2. Onis $M$, Frongillo EA, Blössner $M$. Is malnutrition declining? An analysis of change in levels of child malnutrition since 1980. Bull World Health Organ 2000;78: I 222-33.

3. Schofield C, Ashworth A. Why have mortality rates for severe malnutrition remained so high? Bull World Health Organ 1996;74:223-9.

4. World Health Organization. Management of severe malnutrition: a manual for physicians and other senior health works. Geneva: World Health Organization; 1999. p.62.

5. Gapalan S. Malnutrition: causes, consequences, and solutions. Nutrition 2000;16: 556-8.

6. Deen JL, Funk M, Guevara VC, Saloojee H, Doe JY, Palmer A, et al. Implementation of $\mathrm{WHO}$ guidelines on management of severe malnutrition in hospital in Africa. Bull World Health Organ 2003;81:237-43.

7. Ashworth A. Treatment of severe malnutrition. J Pediatr Gastroenterol Nutr 200 I;32: 516-8.

8. Alam NH, Hamadani JD, Dewan N, Fuchs G. Efficacy and safety of a modified oral rehydration solution (RESOMAL) in the 
treatment of severely malnourished children with watery diarrhea. J Pediatr 2003; 143: 6|4-9.

9. Jellife DB. Evaluacion del estado de nutrición de la comunidad. Ginebra: Organizacion Mundial de la Salud; 1968. (Série de Monografias, 53).

10. World Health Organization. Physical status: The use and interpretation of anthropometry. Geneva: World Health Organization; 1995. p.452. (Technical Report Series, 854).

I I. Monte CGM, Sarni RS. Tratamento hospitalar da criança gravemente desnutrida. In: Departamento de Nutrição da Sociedade Brasileira de Pediatria. Temas de nutrição em pediatria. São Paulo; 2002. p.24-50, n.2.

12. Sarni RS, Munekata RV. Terapia nutricional na desnutrição energético-protéica grave. In: Lopez FA, Sigulem DM, Taddei JAAC. Fundamentos da terapia nutricional em pediatria. São Paulo: Sarvier; 2002. p. I I 5-32.

13. Queiroz SS, Sarni RS, Torres MAA. Carências nutricionais. In: Lopez FA, Brasil ALD. Nutrição e dietética em clínica pediátrica. São Paulo: Atheneu; 2003.

14. Irving S, Simone SD, Hicks F, Verger JT. Nutrition for the critically ill child: enteral and parenteral support. AACN Clin Issues 2000; 1 1:541-58.

15. Nóbrega FJ, Campos D. Distúrbios nutricionais e fraco vínculo mãe-filho. Rio de Janeiro: Revinter; 1996.

16. Ashworth A, Schofield C. Latest developments in the treatment of severe malnutrition in children. Nutrition 1998; | 4:244-5.

17. Prudhon C, Golden MH, Briend A, Mary JY. A model to standardize mortality of severely malnourished children using nutritional status on admission to therapeutic feeding centers. Eur J Clin Nutr 1997;51:77। -7.

18. Perra A, Costello AM. Efficacy of outreach nutrition rehabilitation centers in reducing mortality and improving nutritional outcome of severely malnourished children in Guinea
Bissau. Eur J Clin Nutr 1995;49:353-9.

19. Ashworth A, Khanum. Cost-effective treatment for severely malnourished children: what is the best approach? Health Policy Plan 1997; | 2: | | 5-21.

20. Berckman DS, Lescano AG, Gilman RH, Lopea SL, Blocak MM. Effects of stunting diarrhoeal disease, and parasitc infection during infancy on cognition in late childhood: a follow-up study. Lancet 2002;359:564-7I.

21. Hoffman DJ, Sawaya AL, Verreschi I, Tucker KL, Roberts SB. Why are nutritionally stunted children at increased risk of obesity? Studies of metabolic rate and fat oxidation in shanty-town children from Sao Paulo, Brazil. Am J Clin Nutr 2000;72:702-7.

22. Yamamoto RM, Leone C. A influência das condições de vida no crescimento de lactentes nascidos com peso insuficiente. Rev Paul Pediatr 2003;21: 1 37-42.

23. Koziel S, Jankowska EA. Birthweight and stature, body mass index and fat distribution of | 4-year-old polish adolescents. J Pediatr Child Health 2002;38:55-8.

24. Pescador MVB, Streher AAF, Silva JMF, Valente GCC, Nakagari M, Boguszewski MCS. Aspectos endocrinológicos das crianças e adultos nascidos pequenos para a idade gestacional. Arq Bras Endocrinol Metab 200 I;45: I5-23

25. Rice Al, Sacco L, Hyder A, Black RE. Malnutrition as an underlying cause of childhood deaths associated with infections diseases in developing countries. Bull World Health Organ;78: | 207-2 I.

26. Man W, Weber M, Palmer A, Schneider G, Wadda R, Jaffar $S$, et al. Nutritional status of children admitted to hospital with different diseases and its relationship to outcome in The Gambia, West Africa. Trop Med Int Health 1998;3:678-86.

27. Falbo AR, Alves JGB. Desnutrição grave: alguns aspectos clínicos, epidemiológicos de crianças hospitalizadas no Instituto Materno Infantil de
Pernambuco (IMIP), Brasil. Cad Saúde Pública 2002; | 8: 1473-7.

28. Golden MH, Ramdath D. Free radicals in the pathogenesis of kwashiorkor. Proc Nutr Soc 1987;46:53-68.

29. Scherbaum V, Furst P. New concepts on nutritional management of severe malnutrition: the role of protein. Curr Opin Clin Nutr Metab 2000;3:31-8.

30. Golden $\mathrm{MH}$. Oedematous malnutrition. $\mathrm{Br}$ Med Bull 1998;54:433-44.

31. Golden MH. Issues in kwashiorkor. Lancet 1994;343:292.

32. Golden MHN. The development of concepts of malnutrition. J Nutr 2002; 132:21 I7S-22S.

33. Nichols BL, Dudley MA, Nichols VN, Putman M, Avery SE, Fraley JK, et al. Effects of malnutrition expression and activity of lactase in children. Gastroenterology 1997; | | 2:742-5 | .

34. Nichols BL, Nichols VN, Putman M, Avery SE, Fraley JK, Quaroni A, et al. Contribution of villous atrophy to reduced intestinal maltase in infants with malnutrition. I Pediatr Gastroenterol Nutr 2000;30:497-502.

35. Kukuruzovic RH, Brewster DR. Milk formulas in acute gastroenteritis and malnutrition: a randomized trial. J Pediatr Child Health 2002;38:57|-7.

36. Crook MA, Hally V, Panteli V. The importance of the refeeding syndrome. Nutrition 2001; 17:632-7.

Artigo recebido: 30/0 / /04 Aceito para publicação: 21/06/04 